\title{
Le sens de la politique et des patients
}

\section{Jürg Schlup}

Dr méd., président de la FMH

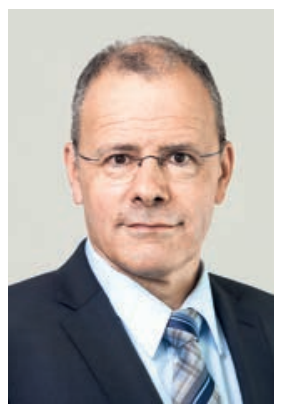

En 2005, lorsque le poste de rédacteur en chef du Bulletin des médecins suisses a été mis au concours, le nom de Bruno Kesseli était si incontournable qu'il paraissait manquer dans le profil d'exigences. Car tout concordait parfaitement. Il avait exercé comme médecin et connaissait la routine d'un hôpital ou d'un cabinet médical. Au fait des défis de notre profession, il connaissait aussi les discussions et les sensibilités autour de la politique de santé et de la politique professionnelle. Sa formation et son expérience de journaliste lui avaient permis d'acquérir les compétences nécessaires à la fois pour le développement d'une revue comme le Bulletin des médecins suisses et pour diriger une rédaction avec professionnalisme.

Le Bulletin des médecins suisses, et au travers de lui, la FMH, toutes ses organisations affiliées et ses membres, ont largement profité des doubles compétences de Bruno Kesseli. Il me semble aussi que ses qualités humaines se sont enrichies du défi personnel de devoir composer avec différents points de vue. Les membres du Comité central de la FMH ont non seulement pu apprécier son ouverture, cordiale et réfléchie, son large sourire communicatif et son empathie, son regard particulièrement large, mais aussi son sens pour les préoccupations des médecins et les défis de la politique. Cette sensibilité l'a aidé à répondre aux aléas quotidiens d'un rédacteur en chef du BMS et à faire face toujours avec pertinence aux situations délicates de la politique professionnelle.

\section{Malgré son double talent, Bruno Kesseli n’aime pas faire les choses à moitié.}

Si Bruno Kesseli est arrivé à donner une voix au BMS tout en offrant habilement une tribune à des opinions divergentes, c'est aussi grâce à la structure claire qu'il a mise en place pour le bulletin jaune: une séparation claire en deux parties. La première reflète l'opinion officielle de la FMH, obtenue après des décisions prises au vote, tandis que la deuxième est libre sur le plan jour- nalistique. Il a pu ainsi maintenir la fonction du BMS en tant qu'organe politique de la FMH, tout en garantissant son indépendance. Distinguer entre positions officielles et voix critiques et les intégrer dans une seule

\section{Nous perdons un excellent rédacteur en chef} pour le BMS, mais la Suisse gagne un médecin de famille passionné!

et même publication s'est avéré être une clé du succès pour le BMS qui reflète, ainsi, la diversité d'opinion du corps médical.

La façon dont Bruno a dirigé le BMS ces treize dernières années, mais aussi ses propres articles, toujours passionnants et bien documentés, montrent qu'il est un journaliste. La poursuite de la médecine en parallèle de son activité pour le BMS et son retour dans un cabinet de généraliste montrent aussi que la profession de médecin est restée plus qu'une simple source de connaissances utiles. Mais surtout cette décision montre que Bruno, malgré son double talent, n'aime pas faire les choses à moitié. Pourtant, tant son équipe de rédaction que son cabinet de groupe avaient la certitude qu'il pouvait être à la hauteur de ces deux fonctions. Mais il a quand même préféré s'en remettre à sa propre échelle d'exigences et se consacre désormais totalement à ses patients.

Cher Bruno, nous te remercions pour toutes ces années d'engagement au service du Bulletin des médecins suisses et du corps médical. La collaboration avec toi a toujours été stimulante et agréable. Tu nous as habitués à la fiabilité de ton instinct pour trouver le meilleur équilibre, nous sommes donc certains que tu as pris pour toi la meilleure décision! Au nom du Comité central de la FMH, je te souhaite le meilleur pour cette prochaine étape, et je complimente tes patients. Ton retour dans un cabinet médical est une perte pour nous mais un gain pour eux. Nous perdons un excellent rédacteur en chef pour le Bulletin des médecins suisses, mais la Suisse gagne un médecin de famille passionné! 